\title{
Traqueostomía Abierta vs Traqueostomía Percutánea
}

\author{
Open vs percutaneous tracheostomy
}

Carlos Celedón L1', Katherine Walker J', Alfredo Naser G', Paola Neumann M¹, Rodolfo Nazar S'1.

\section{RESUMEN}

Introducción: Latraqueostomía estáindicadaparaprevenir el daño laríngeo producido por intubaciones prolongadas, mejorar la higiene traqueal, disminuir el espacio muerto y minimizar la estadía en Unidades de Pacientes Oríticos (UPC). La técnica más utilizada es la abierta, que permite un adecuado control de la anatomía y de la hemostasia Actualmente, en las UPCes cada vez más habitual, la realización de la técnica percutánea Varios trabajos indican que es una técnica rápida y con menos complicaciones, pero aún existe controversia

Objetivo: Comparar a través de un estudio prospectivo, ambas técnicas en cuanto a duración del procedimiento y complicaciones peri y postoperatorias.

Material y método: De un total de 91 pacientes, se seleccionaron 50 que no tenían contraindicación para realizar técnica percutánea En esos 50 pacientes se realizó en forma aleatoria, técnica abierta y técnica percutánea obteniendo 2 grupos de 25 pacientes (Grupos A y P). Todos los pacientes fueron intervenidos en pabellón y bajo anestesia general.

Resultados: La duración total del procedimiento incluida la anestesia fue de 65,8 min y de 59,2 min y la duración de la cirugía fue de 40,4 min y 32,2 min, respectivamente. Las complicaciones perioperatorias fueron: grupo $A 16 \%$ (desaturación y sangrado lo más frecuente) y Grupo P: $40 \%$ (desaturación y pérdida de vía aérea lo más frecuente) y las posoperatorias de $8 \%$ y $12 \%$ (sangrado e infección lo más frecuente en ambos grupos) en los grupos $A$ y $P$, respectivamente.

Conclusión: Al comparar ambas técnicas, no hubo en nuestras manos diferencias estadísticamente significativas en cuanto al número de complicaciones ni en cuanto a la duración del procedimiento.

Palabras Clave: Traqueostomía abierta, traqueostomía percutánea, complicaciones.

\begin{abstract}
Introduction: Tracheostomy is indicated to prevent laryngeal injury in cases of long-term translaryngeal intubation, to improve tracheal hygiene, decrease the dead space and reduce the time spent in intensive care units (IO). The open technique is the most commonly used technique, which allows for an adequate control of anatomy and haemostasis. Ourrently, the percutaneous technique is increasingly used in CPUs, since it is a fast technique, with less perioperative complications. However, there is still controversy on this issue.

Aim: This paper is a prospective study, designed to compare both techniques in terms of procedure duration and peri- and postoperative complications.

Material and method: Out of a total of 91 patients, 50 were selected that did not have any counterindications for the percutaneous technique. These 50 patients were randomly assigned to either the open or the percutanous technique groups, obtaining 2 groups of 25 patients
\end{abstract}

Médico, Servicio de Otorrinolaringología, Hospital Cínico Universidad de Chile. 
(Groups A and P). All patients had the surgery performed in the operating room, under general anesthesia

Results: Total duration of the procedure (including anesthesia) was 65.8 and $59.2 \mathrm{~min}$, and surgery duration was 40.4 and $32.2 \mathrm{~min}$, respectively. Perioperative complications were: Group A, 16\% (oxygen desaturation and bleeding were the more frequent problems), and Goup $P, 40 \%$ (oxygen desaturation and airway loss were the more frequent problems). Postoperative complications in Groups $A$ and $P$ were 8 and 12\% (bleeding and infection being the more frequent problems in both groups), respectively.

Conclusion: In our study, a comparison of both techniques did not show significant differences regarding either number of complications or duration of the procedure.

Key words: Open tracheostomy, percutaneous tracheostomy, complications.

\section{INTRODUCCIÓN}

La traqueostomía es un procedimiento quirúrgico frecuente en nuestro medio, especialmente indicado en los pacientes de las Unidades de Cuidados Intensivos (UCl) sometidos a ventilación asistida. Su utilidad radica en la prevención de daño laríngeo provocado por intubaciones prolongadas, especialmente en aquellas que persisten por más de 10 días, presentándose entre $1 \%$ y $19 \%$ de complicaciones a largo plazo, destacando las estenosis laringotraqueales ${ }^{1}$ y hasta $94 \%$ de daño histológico ${ }^{2}$. Otra ventaja de la traqueostomía es que disminuye la duración de la ventilación asistida y la estadía en UA, minimizando el riesgo de adquisición de infecciones intrahospitalarias ${ }^{3}$.

Los primeros registros que existen de este procedimiento son de hace por lo menos 3000 años$^{4}$. Desde esa fecha hasta hoy la técnica fue difundida, pasando inicialmente por períodos en que las complicaciones eran altísimas. En el año 1800 Trousseau fue celebrado por lograr $25 \%$ de sobrevida al procedimiento realizado en niños con difteria5. Recién en 1909, Chevalier Jackson estandariza la técnica y reduce el porcentaje de complicaciones a $2 \%{ }^{6}$.

La técnica clásica es la traqueostomía subcricoidea que se realiza en los anillos traqueales proximales $\left(2^{\circ}-3^{\circ}\right)$. Sus ventajas incluyen mayor exposición anatómica y control de hemostasia En 1953, Seldinger describió la técnica de cateterización vascular con guía percutánea7. Esta técnica se adaptó a la tráquea surgiendo la primera tra queostomía percutánea moderna en 1955, reportada por Sheldon y col${ }^{8}$. En 1985, Caaglia y col estandarizan esta nueva técnica «fácil, rápida y susceptible de ser realizada en la cama del pacien- te»?. Esta técnica consiste en la introducción de una guía bajo visión endoscópica seguida por la introducción de dilatadores seriados hasta llegar a la instalación del tubo endotraqueal. Existen publica ciones que describen mayor tasa de complicaciones perioperatorias y de mayor severidad en la técnica percutánea; pero mayor número de complicaciones posoperatorias en la técnica abierta ${ }^{10}$; en cambio otros defienden la técnica percutánea argumentando menor número de complicaciones ${ }^{11,12}$ como son la infección posoperatoria y el sangrado periostomal.

La técnica percutánea poseía cuando recién fue descrita una serie de contraindicaciones ${ }^{7,13,14}$; éstas han ido disminuyendo en forma progresiva a medida que se realizan trabajos que echan por tierra las iniciales aprehensiones. La existencia de estas contraindicaciones hace de la técnica percutánea una técnica destinada a un grupo seleccionado de pacientes, que no podrían compararse con los pacientes sometidos a técnica abierta. Por esta razón en nuestro trabajo se seleccionan las traqueotomías abiertas sin contraindicaciones de técnica percutánea para tener grupos comparables.

En general, en la literatura, se analizan ambas técnicas en estudios retrospectivos comparando la «técnica percutánea en la cama del paciente» con la «técnica abierta realizada en pabellón» ${ }^{15,16}$, esto hace que no sean grupos comparables.

Debido a la escasez de literatura nacional en este rubro y a la ausencia de evidencia clara en la literatura internacional que apoye claramente una y otra técnica, nos planteamos como objetivo comparar ambas técnicas quirúrgicas en dos grupos equivalentes de pacientes, que poseen en común la ausencia de contraindicaciones para traqueostomía percutánea, operados bajo las mismas condiciones 
(en pabellón bajo anestesia general) evaluando la duración del procedimiento y la frecuencia de complicaciones peri y posoperatorias.

\section{MATERIAL Y MÉTODO}

Incluye a todos los pacientes sometidos a traqueostomía en el Hospital Cínico de la Universidad de Chile entre agosto 2001 y septiembre 2004.

De un total de 91 traqueostomías realizadas duranteel período en cuestión, se consideraron 50 pacientes que no tenían contraindicación de técnica percutánea A estos pacientes les fue asignada en forma al eatoria la técnica de traqueostomía a realizar. Así obtuvimos 2 grupos de estudio: el grupo A que corresponde a los pacientes sometidos a técnica abierta y el grupo $\mathrm{P}$ que incluye a los pacientes que fueron sometidos a técnica percutánea Ambos grupos de 25 pacientes cada uno.

Se aplicó a cada uno de ellos un protocolo estándar, previo consentimiento informado, donde se consignaban: datos generales del paciente; la indica ción de ventilación mecánica si correspondía; laindica ción detraqueostomía; tipo de técnicaa realizar; escore Apache; duración de intubación; uso de antibióticos; complicaciones de traslado perioperatorias y posope ratorias; y duración de la cirugía Las complicaciones perioperatorias consignadas fueron: pérdida de vía aérea, arritmias, desaturación $<90 \%$, daño traqueal, muerte; hipotensión: Presión arterial media (PAM) <60, falsa vía, sangrado e incapacidad de terminar el procedimiento. Las complicaciones posoperatorias consignadas fueron infección de herida operatoria, neumotórax, decanulación, sangrado, enfisemasubcutáneo, muerte y dehiscencia de sutura

Las contraindicaciones de la técnica percutánea fueron: cuello corto (rigidez cervical, dificultad a la hiperextensión cervical), pérdida de reparos anatómicos, masas cervicales anteriores, inestabilidad hemodinámica, infección local, cicatriz previa, coagulopatía, edad menor a 10 años.

Como una forma de poder comparar y homologar a todos los pacientes de esta investigación, de acuerdo a su gravedad clínica, se les calculó el índice de gravedad Apache II.

Todas las traqueostomías del grupo en estudio (grupo A y P) fueron realizadas bajo anestesia general en pabellón a objeto de poder analizar los resultados con las menos variantes posibles. Latécnicaabiertafue realizada por miembros del equipo médico de nuestro servicio, o por becados bajo supervisión directa de un médico y corresponde a una técnica estandarizada por el Servicio de ORL del Hospital Cínico de la Universidad de Chile ${ }^{17,18}$. La técnica percutánea fue realizada siempre por el mismo equipo de trabajo. Se utilizó un kit de dilatadores progresivos marca Cook código $\mathrm{G}$ PTS-100-HCy en estos pacientes se utilizaron cánulas con endocánulamarca TRACOEdistribuido por Simeck código 300-07, 300-08 y 300-09.

\section{Técnica percutánea: Descripción de técnica}

Paciente con cuello en hiperextensión. Marca de sitio a puncionar en el punto medio entre escotadura yugular y cartílago cricoides. Infiltración con solución de lidocaína al $2 \%$ más adrenalina en concentración de 1:150.000. Incisión transversal de $1 \mathrm{~cm}$ con bisturí hoja №15 en el mismo punto. Retiro del tubo endotraqueal a nivel subglótico. Nasofibroscopía Punción con trocar bajo visión fibroscópica Introducción de la guía metálica através del trocar, seguido de retiro del trocar. Introducción de dilatadores de diámetros sucesivos. Instalación utilizando la guía, de tubo endotraqueal № 7, 8 ó 9, de acuerdo al lumen traqueal.

\section{Análisis Estadístico}

日 análisis estadístico fue realizado en la Unidad de Estadística del Hospital Cínico de la Universidad de Chile. Los métodos utilizados fueron $t$ student para las variables cuantitativas y $c^{2} y$ test de Fisher para las variables cualitativas.

\section{RESULTADOS}

De un total de 91 pacientes, 41 pacientes tenían contraindicación de técnica percutánea, por lo tanto no fueron considerados. Los 50 pacientes restantes constituyeron nuestros 2 grupos de estudio.

\section{Grupo A (técnica abierta)}

Veinticinco pacientes: este grupo estuvo conformado por 18 mujeres y 7 hombres, con un prome dio de edad de 63 años y un rango que variaba 
Tabla 1. Descripción de grupo de estudio

\begin{tabular}{|lccccccc|}
\hline \multirow{2}{*}{ Sexo } & \multicolumn{2}{c}{ TQT abierta } & \multicolumn{2}{c}{ TQT percutánea } & & & \\
& $N$ & $\%$ & $N$ & $\%$ & $X^{2}$ & $P$ & \\
\hline $\begin{array}{l}\text { Masculino } \\
\text { Femenino }\end{array}$ & 18 & 72 & 13 & 52 & 1,358 & 0,244 & NS \\
Uso ATB & 7 & 28 & 12 & 48 & & & \\
$\quad$ Si & 22 & 88 & 23 & 92 & & 1 & NS \\
No & 3 & 12 & 2 & 8 & & & \\
& Media & D.S. & Media & D.S. & T & P & \\
& & & & & & & \\
Edad & 62,9 & 16 & 55 & 17,3 & 1,676 & 0,1 & NS \\
Días intubación & 18,9 & 9,3 & 23,6 & 12 & $-1,548$ & 0,13 & NS \\
Apache II & 13,4 & 7,9 & 9,9 & 4,2 & 1,95 & 0,06 & NS \\
\hline
\end{tabular}

entre 27 y 85 años. 日 promedio de días de intubación previo a la cirugía fue de 18,9 días.

Veintidós de los 25 pacientes recibía antibiótico al momento de realizar la cirugía y el promedio de score Apache II fue de 13,5.

\section{Grupo P (técnica percutánea)}

Veinticinco pacientes: en este grupo existían 13 mujeres y 12 hombres. 1 promedio de edad fue de 55 años con un rango que variaba entre 19 y 85 años. $日$ promedio de días de intubación previa fue de 23,6 días. Veintitrés de los 25 pacientes recibía antibióticos al momento de realizar la traqueostomía. 日 promedio de score Apache II fue de 9,9 (Tabla 1).

Laindicación detraqueostomíaen $80 \%$ fueventila ción mecánica prolongada, $21 \%$ vía aérea difícil y 5\% higienetraqueal. Existía a veces más de unaindicación.

Las indicaciones de intubación y ventilación mecánica fueron: $62 \%$ neumonía, $20 \%$ accidente vascular encefálico, $12 \%$ causa cardiovascular, $12 \%$ sepsis, $8 \%$ neuropatía (Tabla 2 ).
Tabla 2. Indicación de ventilación mecánica

\begin{tabular}{|lcc|}
\hline & N & $\%$ \\
\hline Neumonía & 47 & 62 \\
AVE & 15 & 20 \\
CV & 9 & 12 \\
Sepsis & 9 & 12 \\
Neuropatía & 6 & 8 \\
Nefropatía & 5 & 7 \\
Pulmonar & 4 & 5 \\
Metabólico & 1 & 1 \\
\hline
\end{tabular}

En cuanto a la duración total del procedimiento, incluida la anestesia, ésta fue de 65,8 minutos en la técnica abierta (grupo A) y de 59,2 minutos en la técnica percutánea (grupo P), las diferencias no fueron estadísticamente significativas. Al evaluar la duración del procedimiento independiente del acto anestésico, se obtuvo que el promedio de tiempo para la realización de la traqueostomía clásica fue de 40,4 minutos versus 32,2 minutos de la técnica percutánea. Esta diferencia tampoco arrojó significancia estadística (Tabla 3).

Tabla 3. Duración de traqueostomía

\begin{tabular}{|lccccccc|}
\hline & \multicolumn{2}{c}{ TQT abierta } & \multicolumn{2}{c}{ TQT percutánea } & & & \\
& X & DS & X & DS & $t$ & $p$ & \\
\hline T. total (min) & 65,8 & 21,8 & 59,2 & 20,24 & 1,109 & 0,27 & NS \\
T. cirugía (min) & 40,4 & 17,3 & 32,2 & 11,91 & 1,95 & 0,06 & NS \\
\hline
\end{tabular}


A revisar la cuantía de sangrado intraoperatorio, se vio que hubo sangrado de moderada cuantía (50 $\mathrm{ml}-100 \mathrm{ml}$ ) en 1 paciente (4\%) del grupo A y en 3 pacientes (12\%) del grupo P. $\mathrm{B}$ resto presentó solamente sangrado leve $(<50 \mathrm{ml})$. Esta diferencia no fue significativa ( $p=0,61$ ).

En cuanto a las complicaciones perioperatorias, 4 de 25 pacientes (16\%) del grupo A presentaron complicaciones. Tres pacientes desaturaron bajo $90 \%$ durante el procedimiento y un paciente presentó sangrado moderado.

En el grupo $P$ se presentaron complicaciones en 10 de 25 pacientes (40\%), las que correspondieron a pérdida de vía aérea en 2 casos, arritmia en 1 caso, que correspondió a fibrilación auricular aguda, desaturación bajo $90 \%$ en 5 casos durante el procedimiento, daño de pared posterior de tráquea en un caso y en un caso hubo que convertir a técnica abierta porqueel paciente poseía una tráquea osificada. Estas diferencias no fueron significativas (Tabla 4).

En cuanto a las complicaciones posoperatorias se presentó solamente infección de herida operatoria en 2 casos (8\%) en el grupo A. En el grupo $\mathrm{P}$ se presentaron complicaciones en $3 \mathrm{pa}$ cientes (12\%), 1 infección de herida operatoria, 1 sangrado y una decanulación accidental. Tampoco hubo significancia estadística (Tabla 5).

En nuestra serie no hubo mortalidad como complicación del procedimiento.

\section{COMENTARIO Y CONCLUSIÓN}

La traqueostomía es un procedimiento ampliamente difundido. $\mathrm{日}$ incremento de los servicios de

Tabla 4. Complicaciones perioperatorias

\begin{tabular}{|lcc|}
\hline & Abierta & Percutánea \\
\hline Desat.OQ (<90\%) & 3 & 5 \\
Pérdida aérea & 0 & 2 \\
Sangrado & 1 & 0 \\
Daño traqueal & 0 & 1 \\
Arritmia & 0 & 1 \\
No finaliza proced. & 0 & 1 \\
& & \\
Total & $4 / 25(16 \%)$ & $10 / 25(40 \%)$ \\
$\chi^{2: 2,48}$ & p: 0,115 & NS \\
\hline
\end{tabular}

medicina intensiva y de pacientes complejos que requieren de ventilación asistida hace que las traqueostomías sean cada vez más habituales. La técnica abierta, fue la primera en ser descrita, y fue la única realizada por muchos años. Pasó por épocas de desprestigio especialmente en sus ini$\operatorname{cios}^{5}$. Esto debido a las altas tasas de complicaciones que culminaban frecuentemente en la muerte. Siguen describiéndose complicaciones entre $2 \%$ y $60 \%{ }^{11,13}$ y mortalidad entre $0 \%$ y $5 \%{ }^{10-12}$; lo que varía de acuerdo al centro, equipo quirúrgico y gravedad del paciente. La técnica percutánea reportada por Sheldon hace ya 50 años $^{9}$, ha sido introducida con éxito. Se presentó como una alternativa a la técnica abierta y es de uso habitual entre los intensivistas, quienes la realizan en la cama del paciente y sin necesidad de un cirujano.

Es muy importante al comparar 2 técnicas evitar cualquier factor que pueda afectar los resultados; es por esto que fuimos cuidadosos en este punto, tratando de homogenizar al máximo los grupos para que fueran comparables tanto en edad, sexo, escore Apache, días de intubación, uso de antibióticos e indicación de traqueostomía, como asimismo las condiciones en que ésta se realizaba. Como la técnica percutánea tiene contraindicaciones ${ }^{7,13,14}$, fue necesario; para igualar ambos grupos de estudio; seleccionar los pacientes para una y otra técnica de un total de pacientes que no presentaran estas contraindicaciones. De esa manera los grupos eran comparables. Fue esa la razón también, por la que se utilizó el mismo ambiente y las mismas condiciones para ambas técnicas: pabellón y anestesia general. La mayoría de los trabajos comparan ambas técnicas con la

Tabla 5. Complicaciones posoperatorias

\begin{tabular}{|lcc|}
\hline & Abierta & Percutánea \\
\hline Infección & 2 & 1 \\
Decanulación & 0 & 1 \\
Sangrado & 0 & 1 \\
Enfisema & 0 & 0 \\
Dehiscencia sutura & 0 & 0 \\
Muerte & 0 & 0 \\
Total & 2/25(8\%) & $3 / 25(12 \%)$ \\
& Fsher & p:1,00 NS \\
\hline
\end{tabular}


siguiente salvedad: seleccionan los pacientes para traqueostomía percutánea de acuerdo a diferentes criterios preestablecidos, pero no ocurre lo mismo con los que son sometidos a cirugía abierta; que por lo general son más complejos. Ora diferencia fundamental al comparar ambas técnicas es que la técnica percutánea se realiza por lo general en la cama del paciente y la abierta se realiza en pabe\|lón ${ }^{13,19,20}$.

Se han realizado múltiples trabajos que defienden una y otra técnica La mayoría analizan en forma aislada una y otra técnica'1,4,15-17, o bien otros las comparan, a nuestro entender en forma no muy rigurosa ${ }^{10-13}$. En un metaanálisis realizado en Stanford, California ${ }^{11}$ se describen complicaciones que fluctúan entre $8 \%$ y $44 \%$, para las traqueostomías percutáneas. Wang en 1992, utilizando la técnica percutánea, describió 3 de 7 pacientes con complicaciones graves, incluida la muerte por lo que finalizó el estudio antes de lo previsto ${ }^{21}$. Nuestras complicaciones perioperatorias fueron de $16 \%$ para la técnica abiertay de $40 \%$ para la técnicapercutánea Esteúltimo grupo presentó complicaciones de mayor gravedad tales como fibrilación auricular aguda, pérdida de vía aérea y daño traqueal que requirió, por seguridad, hacer una esofagoscopía de revisión, que resultó ser normal. Esta mayor gravedad en las complicaciones de la técnica percutánea es descrita en el metaanálisis de Dulguero ${ }^{10}$, quien menciona los eventos cardiorrespiratorios y la muerte como complicaciones más frecuentes en esta técnica que en la técnica abierta Aro trabajo que muestra complicaciones graves es el de Ayoub, de Gran Bretaña que menciona una laceración del arco aórtico con esta técnica a pesar del control endoscópico"2.

Las complicaciones posoperatorias fueron para la técnica abierta y percutánea de $8 \%$ y $12 \%$, respectivamente, siendo el sangrado y la decanulación accidental las que marcan la diferencia y la infección el denominador común. Estaúltima complicación fue más frecuente en la técnica abierta, hecho que también concuerda con la literatura ${ }^{19}$. $日$ sangrado y decanulación como complicaciones posoperatorias más frecuentes en la técnica percutánea también concuerdan con lo descrito ${ }^{11,13}$. No hubo en nuestro trabajo diferencias estadísticamente significativas entre ambas técnicas, al comparar las complicaciones peri y posoperatorias, a pesar de que a simple vista parece que las complicaciones perioperatorias y posoperato- rias en la técnica percutánea fueron más frecuentes y las perioperatorias fueron de mayor gravedad. Se describe claramente una curva de aprendizaje en la técnica percutánea, directamente relacionada con la probabilidad de complicaciones intraoperatorias $^{14,16,19}$. Esto se vería minimizado con la supervisión de un otorrinolaringólogo con experiencia y con la disponibilidad de visión fibroscópica durante el procedimiento ${ }^{16}$. En nuestro trabajo, la traqueostomía percutánea fue realizada por el mismo equipo siempre y bajo visión fibroscópica, tratando justamente de minimizar al máximo este factor. Debe hacerse notar que la experiencia en técnica percutánea se inició durante este trabajo, hecho que puede afectar la frecuencia de complicaciones presentes.

En cuanto a la duración del procedimiento, se describe en la literatura un rango bastante amplio de valores que fluctúa entre 6 y 39 minutos ${ }^{11,15,16,19,23,24}$ para la técnica percutánea y entre 10 y 100 minutos $^{16,19,23}$ para la técnica abierta. Nuestro tiempo fue de 32 minutos para la técnica percutánea y para la técnica abierta fue de 40 minutos, pero esta diferencia no fue significativa. Esto varía obviamente de acuerdo a la experiencia del equipo de trabajo.

En suma podemos concluir que en nuestras manos no existen diferencias estadísticamente significativas entre ambas técnicas en cuanto a duración del procedimiento ni tampoco en cuanto a complicaciones peri y posoperatorias.

\section{Agradecimientos}

- Este estudio clínico es de tipo aleatorio prospectivo, fue aprobado por Comité de Ética del Hospital Clínico Universidad de Chile y forma parte de un Proyecto de Investigación concursado y financiado por la Sociedad Chilena de Otorrinolaringología, Medicina y Cirugía de Cabeza y Quello.

- Sr. Samuel Ávila Malverde. Jefe Servicio de Estadística, Hospital Cínico de la Universidad de Chile.

\section{BIBLIOGRAFÍA}

1. HerNer JE Tracheotomy: Indications and timing. Respir Care 1999, 44: 807-15. 
2. COLICE GL, Stuke TA, DaIN B. Laryngeal complications of prolonged intubation. Chest 1989, 98: 877-84.

3. Grimtins J, Barber V, Morgan L, Young D. Systematic review and meta-analysis of studies of timing of tracheostomy in adult patients undergoing artificial ventilation. BMJ 2005; 330: $1243-7$.

4. Frost EAM. Tracing the tracheostomy. Ann Otol 1976; 85: 618-24.

5. EAVEY RD. The history of tracheotomy. In Tracheotomy: Airway management, Communication and swallowing. Edited by Myers $\mathrm{EN}$, Johnson J., Murry T. San Diego: Singular; 1998; 1-8.

6. JaCkson C. Tracheostomy. Laryngoscope 1909; 19: 285-90.

7. Seldinger SI. Catheter replacement of the needle in percutaneous arteriography. Acta Radiol 1953; 39: 368-76.

8. Shedon CH, Pudenz RH, Freshmattr DB et al. New method for tracheotomy. $J$ Neurosurg 1955; 12: 428-31.

9. Gagla P, Firsching $R$, Syniec C. Eective percutaneous dilational tracheostomy. Chest 1985; 87: 715-9.

10. Dulgugrov P, Grsin C, Pegnegr TV et al. Percutaneous or surgical tracheostomy: a metaanalysis. Crit Care Med 1999; 27: 1617-25.

11. Oreng E, FE WE JR. Dilational versus standard tracheostomy: a meta-analysis. Ann Otol Rhinol Laryngol 2000; 109: 803-7.

12. Frmuan BD, Isabela $K$, Lin $N$ et al. A metaanalysis of prospective trials comparing percutaneous and surgical tracheostomy in critically ill patients. Chest 2000; 118: 1412-18.

13. Massick D, YaO S, Powel D, Giesen D. Bedside Tracheostomy in the intensive Care Unit: A Prospective Randomized Trial comparing Open Surgical Tracheostomy with Endoscopically Guided Percutaneous Dilational Tracheotomy. Laryngoscope 2001; 111: 494-500.
14. Massick D, Powel D, Price P. Quantification of the learning curve for percutaneous dilatational tracheotomy. Laryngoscope 2000; 110: 222-8.

15. Moe K, Sohmid S, Stoeckl S, Wermullar E Percutaneous tracheostomy: A Comprehensive Evaluation. Ann Otol Rhinol Laryngol 1999; 108: 384-91.

16. Donaldson D, Emami A, Wax M. Endoscopically Monitored Percutaneous Dilational Tracheotomy in a Residency Program. Laryngoscope 2000; 110: 1142-6.

17. Naser G, Cegmón C, Neumann M, Oueda JP. Revisión de la traqueostomía percutánea Rev Otorrinolaringol Or Cabeza Ouello 2001; 61: 118-24.

18. Nastr G, Oemeón C, Nazar Ret al. Evaluación de la técnica clásica de traqueostomía Rev Otorrinolaringol Or Cabeza Ouello 2002; 62: 251-4.

19. Friegman Y, FlLdes J, Mizook B. Comparison of percutaneous and surgical tracheostomies. Chest 1996; 110/2: 480-5.

20. Fradis M, Malatskey S, Dor I et al. Early complications of tracheostomy performed in the operating room. J Aolaryngol 2003; 32(1): 55-7.

21. Wang M, Betke G Ward P, Calcatgra T, Watts D. Early Experience with percutaneous tracheotomy. Laryngoscope 1992; 102: 15762.

22. Ayour MO, GRIIfTHS MV. Aortic arch laceration: a lethal complicaction after percutaneous tracheostomy. Laryngoscope 2007; 117(1): 176-8.

23. Maccallum P, Parnes L, Sharpe M, Harris C. Comparison of open, percutaneous and translaryngeal tracheostomies. Otolaryngol Head Neck Surg 2000; 122: 686-90.

24. Kaylie DM, AnDersen PE, Wax MK. An analysis of time and staff utilization for open versus percutaneous tracheostomies. Otolaryngol Head Neck Surg 2003; 128(1): 109-14.

\footnotetext{
Dirección: Dr. Carlos Celedón L.

Luis Thayer Ojeda 0115. Oficina 705. Providencia. Fono: 2322758

Email: celedonl@mi.cl
} 